\title{
Fokker-Planck equation with fractional coordinate derivatives
}

\author{
Vasily E. Tarasov ${ }^{\mathrm{a}, \mathrm{b}, *}$, George M. Zaslavsky ${ }^{\mathrm{a}, \mathrm{c}}$ \\ a Courant Institute of Mathematical Sciences, New York University, 251 Mercer Street, New York, NY 10012, USA \\ b Skobeltsyn Institute of Nuclear Physics, Moscow State University, Moscow 119991, Russia \\ ${ }^{\mathrm{c}}$ Department of Physics, New York University, 2-4 Washington Place, New York, NY 10003, USA
}

\section{A R T I C L E I N F O}

\section{Article history:}

Received 5 May 2008

Received in revised form 5 August 2008

Available online 30 August 2008

\section{Keywords:}

Fractional kinetics

Fractional derivatives

Long-range interaction

Fokker-Planck equation

Kolmogorov-Feller equation

\begin{abstract}
A B S T R A C T
Using the generalized Kolmogorov-Feller equation with long-range interaction, we obtain kinetic equations with fractional derivatives with respect to coordinates. The method of successive approximations, with averaging with respect to a fast variable, is used. The main assumption is that the correlation function of probability densities of particles to make a step has a power-law dependence. As a result, we obtain a Fokker-Planck equation with fractional coordinate derivative of order $1<\alpha<2$.
\end{abstract}

(C) 2008 Elsevier B.V. All rights reserved.

\section{Introduction}

In studying processes with fractal time and long-term memory a generalized kinetic equation was proposed in Ref. [1]. While the equation was of the master equation type, its main property was the presence of the power-type kernel for a probability density to make a step. This type of equation was compared with the Kolmogorov-Feller equation in Ref. [2]. In this paper, we would like to go farther and to show the conditions under which one can obtain a fractional generalization of the Fokker-Planck equation from the Kolmogorov-Feller equation.

Fractional calculus [3-5] has found many applications in recent studies in mechanics and physics, and the interest in fractional equations has been growing continually during the last years [6-12]. Fractional Fokker-Planck equations with coordinate and time derivatives of non-integer order has been suggested in Ref. [13]. The solutions and properties of these equations are described in Refs. [2,8]. The Fokker-Planck equation with fractional coordinate derivatives was also considered in Refs. [14-18].

The Kolmogorov-Feller equation is an integro-differential one, and it belongs to the type of master equations broadly used in different physical applications. It is well-known that the Kolmogorov-Feller equation can lead us to the Fokker-Planck equation [19], under some conditions. In this paper, we use the method of successive approximations with averaging with respect to a fast variable [20]. We suppose that the correlation function of probability densities, which are used in the Kolmogorov-Feller equation, is a power type. The Fokker-Planck equations with coordinate derivatives of order $1<\alpha<2$ are derived.

In Section 2, the Kolmogorov-Feller equation for the one-dimensional case is considered to fix notations and provide convenient references. We note that power-law probability to make a step gives the equation with a fractional derivative. In Section 3, we present a generalization of the Kolmogorov-Feller equation for the two-dimensional case. The method of successive approximations is used for this generalized equation in Section 4. In Section 5, we use averaging with respect to the fast variable, to derive fractional Fokker-Planck equations. Finally, a short conclusion is given in Section 6.

\footnotetext{
* Corresponding author at: Courant Institute of Mathematical Sciences, New York University, 251 Mercer Street, New York, NY 10012, USA.

E-mail address: tarasov@theory.sinp.msu.ru (V.E. Tarasov).
} 


\section{Kolmogorov-Feller equation for one-dimensional case}

\subsection{Operator representation of the KF-equation}

Let $P(t, x)$ be a probability density to find a particle at $x$ at time instant $t$. The normalization condition for $P(t, x)$ is

$$
\int_{-\infty}^{+\infty} \mathrm{d} x P(t, x)=1 \quad(t>0)
$$

The Kolmogorov-Feller (KF) equation has the form

$$
\frac{\partial P(t, x)}{\partial t}=\int_{-\infty}^{+\infty} \mathrm{d} x^{\prime} w\left(x^{\prime}\right)\left[P\left(t, x-x^{\prime}\right)-P(t, x)\right], \quad P(0, x)=\delta(x),
$$

where $w\left(x^{\prime}\right)$ is the probability density of particle to make a step of the length $x^{\prime}$, and

$$
\int_{-\infty}^{+\infty} \mathrm{d} x^{\prime} w\left(x^{\prime}\right)=1
$$

Let us introduce the operator representation of the KF-equation. We define the translation operator

$$
T_{x^{\prime}}=\exp \left\{-x^{\prime} \partial_{x}\right\}
$$

such that

$$
T_{x^{\prime}} P(t, x)=P\left(t, x-x^{\prime}\right),
$$

and the finite difference operator

$$
\Delta_{x^{\prime}}=I-T_{x^{\prime}},
$$

where $I$ is an identity operator. Then the Kolmogorov-Feller equation (1) can be presented as

$$
\frac{\partial P(t, x)}{\partial t}=L(\Delta) P(t, x) .
$$

Here we use the integro-differential operator

$$
L(\Delta)=-\int_{-\infty}^{+\infty} \mathrm{d} x^{\prime} w\left(x^{\prime}\right) \Delta_{x^{\prime}}
$$

The operator (7) will be called the Kolmogorov-Feller operator. Note that power-law probability $w\left(x^{\prime}\right)$ in Eq. (1) allows us to introduce a derivative of non-integer order [4].

\subsection{KF-equation with fractional coordinate derivative}

The well-known fractional Caputo derivative [4] of order $\alpha$ is defined by

$$
{ }^{c} D_{x}^{\alpha} P(x)=\frac{1}{\Gamma(1-\alpha)} \int_{-\infty}^{x} \frac{\mathrm{d} z}{(x-z)^{\alpha}} \frac{\partial P(z)}{\partial z}, \quad(0<\alpha<1) .
$$

The fractional Marchaud derivative [4] of order $\alpha$ is defined by

$$
\mathbf{D}_{x}^{\alpha} P(x)=\frac{1}{\Gamma(-\alpha)} \int_{-\infty}^{x}[P(z)-P(x)] \frac{\mathrm{d} z}{(x-z)^{\alpha+1}}, \quad(0<\alpha<1) .
$$

Using $x^{\prime}=x-z$, Eq. (9) has the form

$$
\mathbf{D}_{x}^{\alpha} P(x)=\frac{1}{\Gamma(-\alpha)} \int_{0}^{\infty} \frac{\mathrm{d} x^{\prime}}{\left(x^{\prime}\right)^{\alpha+1}}\left[P\left(x-x^{\prime}\right)-P(x)\right] .
$$

If the function $w\left(x^{\prime}\right)$ in the KF-equation (1) is the exponential function up to a small parameter $\varepsilon$ such that

$$
w\left(x^{\prime}\right)=\frac{a}{x^{\prime \alpha+1}} H\left(x^{\prime}\right)+O(\varepsilon),
$$

where $H\left(x^{\prime}\right)$ is a Heaviside step function, then Eq. (1) can be presented as

$$
\frac{\partial P(t, x)}{\partial t}=a \mathbf{D}_{x}^{\alpha} P(t, x)+O(\varepsilon), \quad P(0, x)=\delta(x), \quad(0<\alpha<1) .
$$

This equation has a fractional coordinate derivative of order $0<\alpha<1$. Note that the function $w(x)$ is a probability density, and it should satisfy the normalization condition (2). 


\subsection{Generalized KF-equation}

In the general case, we can suppose that probability density of a particle to make a step of the length $x^{\prime}$ depends on the time instant $t$ and the coordinate $x$. Then we should replace $w\left(x^{\prime}\right)$ by $w\left(t, x \mid x^{\prime}\right)$ in KF-equation (1). As a result, we can consider the equation

$$
\varepsilon^{-1} \partial_{t} P(t, x)=\varepsilon \int_{-\infty}^{+\infty} \mathrm{d} x^{\prime} w\left(t, x \mid x^{\prime}\right)\left[P\left(t, x-x^{\prime}\right)-P(t, x)\right], \quad P(0, x)=\delta(x),
$$

where $\varepsilon$ is a small parameter and $\partial_{t}=\partial / \partial t$. Note that the interpretation of the appearance of the small parameter $\varepsilon$ is connected with the change of the scale $t \rightarrow \varepsilon t$, such that $\partial_{t} \rightarrow \varepsilon \partial_{t}$. The function $w\left(t, x \mid x^{\prime}\right)$ is the probability density to make a step of the length $x^{\prime}$ at the time instant $t$ from the coordinate $x$. If $w\left(t, x \mid x^{\prime}\right)=w\left(x^{\prime}\right)$, then Eq. (12) gives Eq. (1). We can assume that during any interval of time $(t, t+\mathrm{d} t)$ the value of the variable $x(t)$ remains equal to $x$, with probability $1-p(t, x) \mathrm{d} t$ and may undergo a change only with probability $p(t, x) \mathrm{d} t$ (see more about this equation in Sec. 55. of Ref. [19]). We suppose that

$$
w\left(t, x \mid x^{\prime}\right)=p(t, x) w\left(x^{\prime}\right),
$$

where $p(t, x)$ is a bounded function. If $p(t, x)=1$, then Eqs. (12) and (13) gives Eq. (1).

Using the operator (5), Eq. (12) can be presented in the operator form

$$
\frac{\partial P(t, x)}{\partial t}=\varepsilon L(t, x, \Delta) P(t, x)
$$

where

$$
L(t, x, \Delta)=-\int_{-\infty}^{+\infty} \mathrm{d} x^{\prime} w\left(t, x \mid x^{\prime}\right) \Delta_{x^{\prime}}
$$

Eq. (14) will be called a generalized KF-equation for one-dimensional case.

\subsection{Successive approximations}

The generalized Kolmogorov-Feller equation (14) can be rewritten in the integral form

$$
P(t, x)-P(0, x)=\varepsilon \int_{0}^{t} \mathrm{~d} \tau L(\tau, x, \Delta) P(\tau, x) .
$$

This equation can be presented as the integral Volterra type equation

$$
P(t, x)=P(0, x)+\varepsilon \int_{0}^{t} \mathrm{~d} t_{1} L\left(t_{1}, x, \Delta\right) P\left(t_{1}, x\right) .
$$

Let us consider the successive approximations. Substitution of Eq. (17) in the form

$$
P\left(t_{1}, x\right)=P(0, x)+\varepsilon \int_{0}^{t_{1}} \mathrm{~d} t_{2} L\left(t_{2}, x \Delta\right) P\left(t_{2}, x\right)
$$

into Eq. (17) gives

$$
P(t, x)=P\left(t_{0}, x\right)+\varepsilon \int_{0}^{t} \mathrm{~d} t_{1} L\left(t_{1}, x, \Delta\right) P(0, x)+\varepsilon^{2} \int_{0}^{t} \mathrm{~d} t_{1} \int_{0}^{t_{1}} \mathrm{~d} t_{2} L\left(t_{1}, x, \Delta\right) L\left(t_{2}, x, \Delta\right) P\left(t_{2}, x\right) .
$$

Changing the variables $t_{1} \rightarrow t_{2}$, and $t \rightarrow t_{1}$ in Eq. (18), and substituting into (19), we obtain an equation up to $\varepsilon^{2}$ in the form

$$
P(t, x)=P(0, x)+\varepsilon \int_{0}^{t} \mathrm{~d} t_{1} L\left(t_{1}, x, \Delta\right) P(0, x)+\varepsilon^{2} \int_{0}^{t} \mathrm{~d} t_{1} \int_{0}^{t_{1}} \mathrm{~d} t_{2} L\left(t_{1}, x, \Delta\right) L\left(t_{2}, x, \Delta\right) P(0, x) .
$$

If the function $w\left(x^{\prime}\right)$ is the exponential function (10), then $L(t, x, \Delta)$ is a differential operator of order $0<\alpha<1$ with respect to $x$, and $L\left(t_{1}, x, \Delta\right) L\left(t_{2}, x, \Delta\right) P\left(t_{2}, x\right)$ is a differential operator of the order $0<2 \alpha<2$. To obtain fractional kinetic equations of the order $0<2 \alpha<2$, we should consider the averaging procedure before a partial differentiation of Eq. (20) with respect to time is realized. Without averaging, we derive an equation of order $0<\alpha<1$. 


\section{Distribution function and Kolmogorov-Feller equation for two-dimensional case}

Let $P(t, x, y)$ be a function of probability distribution in a phase space. The variables $x$ and $y$ describe the phase space of a system. There are the following interpretations for the variables $x$ and $y$.

- A system with one degree of freedom can be presented by momentum $x=p$, and coordinates $y=q$.

- A system can be described by action $x=I$, and phase $y=\theta$.

- $n$-particle system can be defined by $x=\left(q_{1}, p_{1}\right)$ and $y=\left(q_{2}, p_{2}, \ldots, q_{n}, p_{n}\right)$, or $x=q_{1}$, and $y=\left(p_{1}, q_{2}, p_{2}, \ldots, q_{n}, p_{n}\right)$.

- The variables $x$ describe a system, and $y$ describes an environment of this system.

We plan to use the reduced distribution and average values with respect to $y$, where $y$ will be considered as a fast variable.

\subsection{Generalized KF-equation for two-dimensional case}

We assume that $x \in X \subset \mathbb{R}$ and $y \in Y \subset \mathbb{R}$, then $\mathbf{r}=(x, y) \in X \times Y \subset \mathbb{R}^{2}$. We plan to consider $x$ as a slow variable and $y$ will be considered as a fast variable. The distribution function $P(t, \mathbf{r})$ in the region $X \times Y$ satisfies the generalized Kolmogorov-Feller equation

$$
\frac{\partial P(t, \mathbf{r})}{\partial t}=\varepsilon \int_{X \times Y} \mathrm{~d}^{2} r_{1} w\left(t, \mathbf{r} \mid \mathbf{r}_{1}\right)\left[P\left(t, \mathbf{r}-\mathbf{r}_{1}\right)-P(t, \mathbf{r})\right],
$$

where $\mathrm{d}^{2} r_{1}=\mathrm{d} x_{1} \mathrm{~d} y_{1}$, and $\varepsilon$ is a small parameter. Here $w\left(t, \mathbf{r} \mid \mathbf{r}_{1}\right)$ is the probability density to make a step $\mathbf{r}_{1}$ at the time instant $t$ from the point $\mathbf{r}$.

Eq. (21) can be presented in the form

$$
\frac{\partial}{\partial t} P(t, \mathbf{r})=\varepsilon L(t, \mathbf{r}, \Delta) P(t, \mathbf{r})
$$

where $L(t, \mathbf{r}, \Delta)$ is a Kolmogorov-Feller operator

$$
L(t, \mathbf{r}, \Delta)=-\int_{X \times Y} \mathrm{~d}^{2} r_{1} w\left(t, \mathbf{r} \mid \mathbf{r}_{1}\right) \Delta_{\mathbf{r}_{1}} .
$$

Here, $\Delta_{\mathbf{r}_{1}}$ is a finite difference operator

$$
\Delta_{\mathbf{r}_{1}}=I-T_{\mathbf{r}_{1}},
$$

where $T_{\mathbf{r}_{1}}$ is a translation operator in $X \times Y$, that is defined by

$$
T_{\mathbf{r}_{1}}=\exp \left\{-\mathbf{r}_{1} \nabla\right\}=\exp \left\{-x_{1} \partial_{x}-y_{1} \partial_{y}\right\}
$$

We can assume that during any interval of time $(t, t+\mathrm{d} t)$ the value of the variable $\mathbf{r}(t)$ remains equal to $\mathbf{r}$ with probability $1-p(t, \mathbf{r}) \mathrm{d} t$ and may undergo a change only with probability $p(t, \mathbf{r}) \mathrm{d} t$ (see Sec. 55. in Ref. [19]). Then,

$$
w\left(t, \mathbf{r} \mid \mathbf{r}^{\prime}\right)=p(t, \mathbf{r}) w\left(\mathbf{r}^{\prime}\right),
$$

where $p(t, \mathbf{r})$ is a bounded function.

We can use the variables $x, y$ instead of $\mathbf{r}$. Then Eq. (21) for the distribution function $P(t, x, y)$ will be presented in the form

$$
\frac{\partial P(t, x, y)}{\partial t}=\varepsilon \int_{X \times Y} \mathrm{~d} x_{1} \mathrm{~d} y_{1} w\left(t, x, y \mid x_{1}, y_{1}\right)\left[P\left(t, x-x_{1}, y-y_{1}\right)-P(t, x, y)\right] .
$$

This equation can be rewritten as

$$
\frac{\partial}{\partial t} P(t, x, y)=\varepsilon L\left(t, x, y, \Delta_{x}, \Delta_{y}\right) P(t, x, y),
$$

where $L\left(t, x, y, \Delta_{x}, \Delta_{y}\right)$ is a Kolmogorov-Feller operator

$$
L\left(t, x, y, \Delta_{x}, \Delta_{y}\right)=\int_{X \times Y} \mathrm{~d} x_{1} \mathrm{~d} y_{1} w\left(t, x, y \mid x^{\prime}, y^{\prime}\right)\left[T_{x_{1}} T_{y_{1}}-I\right] .
$$

This is the generalized Kolmogorov-Feller equation in the operator form. 


\section{Method of successive approximations}

The generalized Kolmogorov-Feller equation

$$
\frac{\partial}{\partial t} P(t, \mathbf{r})=\varepsilon L(t, \mathbf{r}, \Delta) P(t, \mathbf{r})
$$

can be presented as the integral Volterra equation

$$
P(t, \mathbf{r})=P_{0}(\mathbf{r})+\varepsilon \int_{0}^{t} \mathrm{~d} t_{1} L\left(t_{1}, \mathbf{r}, \Delta\right) P\left(t_{1}, \mathbf{r}\right),
$$

where $P_{0}(\mathbf{r})=P(0, \mathbf{r})$. Substitution of Eq. (29) in the form

$$
P\left(t_{1}, \mathbf{r}\right)=P_{0}(\mathbf{r})+\varepsilon \int_{0}^{t_{1}} \mathrm{~d} t_{2} L\left(t_{2}, \mathbf{r}, \Delta\right) P\left(t_{2}, \mathbf{r}\right)
$$

into Eq. (29), gives

$$
P(t, \mathbf{r})=P_{0}(\mathbf{r})+\varepsilon \int_{0}^{t} \mathrm{~d} t_{1} L\left(t_{1}, \mathbf{r}, \Delta\right) P_{0}(\mathbf{r})+\varepsilon^{2} \int_{0}^{t} \mathrm{~d} t_{1} \int_{0}^{t_{1}} \mathrm{~d} t_{2} L\left(t_{1}, \mathbf{r}, \Delta\right) L\left(t_{2}, \mathbf{r}, \Delta\right) P\left(t_{2}, \mathbf{r}\right) .
$$

Changing the variables $t_{1} \rightarrow t_{2}$, and $t \rightarrow t_{1}$ in Eq. (30), and substituting into (31), we get

$$
P(t, \mathbf{r})=P_{0}(\mathbf{r})+\varepsilon \int_{0}^{t} \mathrm{~d} t_{1} L\left(t_{1}, \mathbf{r}, \Delta\right) P_{0}(\mathbf{r})+\varepsilon^{2} \int_{0}^{t} \mathrm{~d} t_{1} \int_{0}^{t_{1}} \mathrm{~d} t_{2} L\left(t_{1}, \mathbf{r}, \Delta\right) L\left(t_{2}, \mathbf{r}, \Delta\right) P_{0}(\mathbf{r})+\cdots .
$$

Using the chronological multiplication

$$
T\left\{L\left(t_{1}, \mathbf{r}, \Delta\right) L\left(t_{2}, \mathbf{r}, \Delta\right)\right\}= \begin{cases}L\left(t_{1}, \mathbf{r}, \Delta\right) L\left(t_{2}, \mathbf{r}, \Delta\right) & t_{1}>t_{2} \\ L\left(t_{2}, \mathbf{r}, \Delta\right) L\left(t_{1}, \mathbf{r}, \Delta\right) & t_{2}>t_{1}\end{cases}
$$

Eq. (32), can be symmetric with respect to $t_{1}$ and $t_{2}$ :

$$
P(t, \mathbf{r})=P_{0}(\mathbf{r})+\varepsilon \int_{0}^{t} \mathrm{~d} t_{1} L\left(t_{1}, \mathbf{r}, \Delta\right) P_{0}(\mathbf{r})+\frac{1}{2} \varepsilon^{2} \int_{0}^{t} \mathrm{~d} t_{1} \int_{0}^{t} \mathrm{~d} t_{2} T\left\{L\left(t_{1}, \mathbf{r}, \Delta\right) L\left(t_{2}, \mathbf{r}, \Delta\right)\right\} P_{0}(\mathbf{r})+\cdots .
$$

This is the symmetric representation of Eq. (32).

\section{Averaging with respect to the fast variable}

In this section, let us consider the variables $\mathbf{r}=(x, y)$ as slow $(x)$ and fast $(y)$. Substitution of (23) into (34), gives

$$
\begin{aligned}
P(t, \mathbf{r})= & P_{0}(\mathbf{r})-\varepsilon \int_{0}^{t} \mathrm{~d} t_{1} \int_{X \times Y} \mathrm{~d}^{2} r_{1} w\left(t_{1}, \mathbf{r} \mid \mathbf{r}_{1}\right) \Delta_{\mathbf{r}_{1}} P_{0}(\mathbf{r}) \\
& +\frac{1}{2} \varepsilon^{2} \int_{0}^{t} \mathrm{~d} t_{1} \int_{0}^{t} \mathrm{~d} t_{2} \int_{X \times Y} \mathrm{~d}^{2} r_{1} \int_{X \times Y} \mathrm{~d}^{2} r_{2} T\left\{w\left(t_{1}, \mathbf{r} \mid \mathbf{r}_{1}\right) \Delta_{\mathbf{r}_{1}} w\left(t_{2}, \mathbf{r} \mid \mathbf{r}_{2}\right) \Delta_{\mathbf{r}_{2}}\right\} P_{0}(\mathbf{r})+O\left(\varepsilon^{3}\right) .
\end{aligned}
$$

The function $w\left(t, \mathbf{r} \mid \mathbf{r}_{1}\right)$ is the probability density to make a step of the vector $\mathbf{r}_{1}$ at the time instant $t$ from the point $\mathbf{r}$. The first assumption, is that this probability density has a weak dependence (up to terms of order $O(\varepsilon)$ ) on the point $\mathbf{r}$, i.e.,

$$
w\left(t, \mathbf{r}+\mathbf{r}_{1} \mid \mathbf{r}_{2}\right)=w\left(t, \mathbf{r} \mid \mathbf{r}_{2}\right)+O(\varepsilon) .
$$

Then

$$
\Delta_{\mathbf{r}_{1}} w\left(t_{2}, \mathbf{r} \mid \mathbf{r}_{2}\right)=0+O(\varepsilon)
$$

and

$$
w\left(t_{1}, \mathbf{r} \mid \mathbf{r}_{1}\right) \Delta_{\mathbf{r}_{1}} w\left(t_{2}, \mathbf{r} \mid \mathbf{r}_{2}\right) \Delta_{\mathbf{r}_{2}}=w\left(t_{1}, \mathbf{r} \mid \mathbf{r}_{1}\right) w\left(t_{2}, \mathbf{r} \mid \mathbf{r}_{2}\right) \Delta_{\mathbf{r}_{1}} \Delta_{\mathbf{r}_{2}}+O(\varepsilon) .
$$

As a result, Eq. (35) has the form

$$
\begin{aligned}
P(t, \mathbf{r})= & P_{0}(\mathbf{r})-\varepsilon \int_{0}^{t} \mathrm{~d} t_{1} \int_{X \times Y} \mathrm{~d}^{2} r_{1} w\left(t_{1}, \mathbf{r} \mid \mathbf{r}_{1}\right) \Delta_{\mathbf{r}_{1}} P_{0}(\mathbf{r}) \\
& +\frac{1}{2} \varepsilon^{2} \int_{0}^{t} \mathrm{~d} t_{1} \int_{0}^{t} \mathrm{~d} t_{2} \int_{X \times Y} \mathrm{~d}^{2} r_{1} \int_{X \times Y} \mathrm{~d}^{2} r_{2} T\left\{w\left(t_{1}, \mathbf{r} \mid \mathbf{r}_{1}\right) w\left(t_{2}, \mathbf{r} \mid \mathbf{r}_{2}\right)\right\} \Delta_{\mathbf{r}_{1}} \Delta_{\mathbf{r}_{2}} P_{0}(\mathbf{r})+O\left(\varepsilon^{3}\right) .
\end{aligned}
$$


The second assumption states that $P_{0}(\mathbf{r})=P_{0}(x, y)$ does not depend on the fast variable $y$ up to $\varepsilon$-terms, such that

$$
P_{0}(\mathbf{r})=P_{0}(x, y)=\rho_{0}(x)+O(\varepsilon) .
$$

Then, we have

$$
\Delta_{\mathbf{r}_{1}} \Delta_{\mathbf{r}_{2}} P_{0}(\mathbf{r})=\Delta_{x_{1}} \Delta_{x_{2}} \rho_{0}(x)+O(\varepsilon) .
$$

Averaging over the variable $y$ will be denoted by \langle\rangle$_{y}$. We also use the notations

$$
\rho(t, x)=\langle P(t, \mathbf{r})\rangle_{y},
$$

and $\rho(0, x)=\rho_{0}(x)$.

Averaging of Eq. (35) with respect to the fast variable $y$, we obtain

$$
\begin{aligned}
\rho(t, x)= & \rho_{0}(x)-\varepsilon \int_{0}^{t} \mathrm{~d} t_{1} \int_{X} \mathrm{~d} x_{1} A\left(t_{1}, x \mid x_{1}\right) \Delta_{x_{1}} \rho_{0}(x) \\
& +\frac{1}{2} \varepsilon^{2} \int_{0}^{t} \mathrm{~d} t_{1} \int_{X} \mathrm{~d} x_{1} \int_{X} \mathrm{~d} x_{2} B\left(t_{1}, x \mid x_{1}, x_{2}\right) \Delta_{x_{1}} \Delta_{x_{2}} \rho_{0}(x)+O\left(\varepsilon^{3}\right),
\end{aligned}
$$

where we introduce the functions

$$
\begin{aligned}
& A\left(t_{1}, x \mid x_{1}\right)=\int_{Y} \mathrm{~d} y_{1}\left\langle w\left(t_{1}, \mathbf{r} \mid \mathbf{r}_{1}\right)\right\rangle_{y}, \\
& B\left(t_{1}, x \mid x_{1}, x_{2}\right)=\int_{0}^{t} \mathrm{~d} t_{2} \int_{Y} \mathrm{~d} y_{1} \int_{Y} \mathrm{~d} y_{2}\left\langle T\left\{w\left(t_{1}, \mathbf{r} \mid \mathbf{r}_{1}\right) w\left(t_{2}, \mathbf{r} \mid \mathbf{r}_{2}\right)\right\}\right\rangle_{y} .
\end{aligned}
$$

Using $\mathbf{r}=(x, y)$, these functions can be presented by

$$
\begin{aligned}
& A\left(t_{1}, x \mid x_{1}\right)=\int_{Y} \mathrm{~d} y_{1}\left\langle w\left(t_{1}, x, y \mid x_{1}, y_{1}\right)\right\rangle_{y}, \\
& B\left(t_{1}, x \mid x_{1}, x_{2}\right)=\int_{0}^{t} \mathrm{~d} t_{2} \int_{Y} \mathrm{~d} y_{1} \int_{Y} \mathrm{~d} y_{2}\left\langle T\left\{w\left(t_{1}, x, y \mid x_{1}, y_{1}\right) w\left(t_{2}, x, y \mid x_{2}, y_{2}\right)\right\}\right\rangle_{y} .
\end{aligned}
$$

The third assumption, is that the function $B\left(t_{1}, x \mid x_{1}, x_{2}\right)$ is diagonal with respect to variables $x_{1}$ and $x_{2}$ up to $\varepsilon$-term, i.e.,

$$
B\left(t_{1}, x \mid x_{1}, x_{2}\right)=B\left(t_{1}, x \mid x_{1}\right) \delta\left(x_{1}-x_{2}\right)+O(\varepsilon) .
$$

Then the operator $\Delta_{x_{1}} \Delta_{x_{2}}$ will be the finite difference operator $\Delta_{x_{1}}^{2}$ of second order. This allows us to have a fractional derivative for the exponential function $B\left(t, x \mid x_{1}\right)$ since Marchaud and Riesz fractional derivatives [3] are defined through the finite difference operator.

The fourth assumption, is that the functions $A\left(t_{1}, x \mid x_{1}\right)$ and $B\left(t_{1}, x \mid x_{1}\right)$ are exponential functions up to $\varepsilon$ such that

$$
\begin{aligned}
& A\left(t, x \mid x_{1}\right)=a(t) \frac{1}{\kappa\left(\alpha_{1}, 1\right)} \frac{1}{\left|x_{1}\right|^{\alpha_{1}+1}} H\left(x_{1}\right)+O(\varepsilon), \quad\left(0<\alpha_{1}<1\right), \\
& B\left(t, x \mid x_{1}\right)=b(t, x) \frac{1}{\kappa\left(\alpha_{2}, 2\right)} \frac{1}{\left|x_{1}\right|^{\alpha_{2}}} H\left(x_{1}\right)+O(\varepsilon), \quad\left(1<\alpha_{2}<2\right),
\end{aligned}
$$

where $H(x)$ is the Heaviside step function, and

$$
\kappa(\alpha, n)=-\Gamma(\alpha) A_{n}(\alpha), \quad A_{n}(\alpha)=\sum_{k=0}^{n}(-1)^{k-1} \frac{n !}{k !(n-k) !} k^{\alpha},
$$

with $n-1<\alpha<n$.

As a result, Eq. (40) gives

$$
\rho(t, x)=\rho_{0}(x)+\varepsilon \int_{0}^{t} \mathrm{~d} t_{1} a\left(t_{1}\right) \mathbf{D}_{x}^{\alpha_{1}} \rho_{0}(x)+\varepsilon^{2} \int_{0}^{t} \mathrm{~d} t_{1} b\left(t_{1}, x\right) \mathbf{D}_{x}^{\alpha_{2}} \rho_{0}(x)+O\left(\varepsilon^{3}\right) .
$$

Here, $\mathbf{D}_{x}^{\alpha}$ is Marchaud fractional derivative [3] of order $\alpha$ with respect to $x$, which is defined by the equation

$$
\mathbf{D}_{x}^{\alpha} f(x)=\frac{1}{\kappa(\alpha, n)} \int_{0}^{\infty} \frac{\Delta_{y}^{n} f(x)}{y^{\alpha+2-n}} \mathrm{~d} y, \quad(n-1<\alpha<n),
$$

where $\Delta_{y}^{n}$ is a finite difference of order $n$ such that

$$
\Delta_{y}^{n} f(x)=\left(I-T_{y}\right)^{n} f(x)=\sum_{m=0}^{n}(-1)^{m} \frac{n !}{m !(n-m) !} f(x-m y) .
$$


In general, the variables $x$ and $x_{1}$ can be vectors in the $N$-dimensional space $\mathbb{R}^{N}$, where $N=1,2,3, \ldots$ The fourth assumption for the functions $A\left(t_{1}, x \mid x_{1}\right)$ and $B\left(t_{1}, x \mid x_{1}\right)$ can be realized in the form other than (46) and (47). We can suppose that the functions $A\left(t_{1}, x \mid x_{1}\right)$ and $B\left(t_{1}, x \mid x_{1}\right)$ are exponential functions up to $\varepsilon$ such that

$$
\begin{aligned}
& A\left(t, x \mid x_{1}\right)=a(t) \frac{1}{d_{N}\left(1, \alpha_{1}\right)} \frac{1}{\left|x_{1}\right|^{N+\alpha_{1}}}+O(\varepsilon), \quad\left(0<\alpha_{1}<1\right), \\
& B\left(t, x \mid x_{1}\right)=b(t, x) \frac{1}{d_{N}\left(2, \alpha_{2}\right)} \frac{1}{\left|x_{1}\right|^{N+\alpha_{2}}}+O(\varepsilon), \quad\left(1<\alpha_{2}<2\right),
\end{aligned}
$$

where $x, x_{1} \in \mathbb{R}^{N}$, and

$$
d_{N}(n, \alpha)=\frac{2^{-\alpha} \pi^{1+N / 2} A_{n}(\alpha)}{\sin (\alpha \pi / 2) \Gamma(1+\alpha / 2) \Gamma((N+\alpha) / 2)}
$$

with $n-1<\alpha<n$. As a result, Eq. (40) gives a fractional equation of the form (49), where $\mathbf{D}_{x}^{\alpha}$ is the fractional Riesz derivative (see Ref. [3] Sec. 25.4) of order $\alpha$ with respect to $x \in \mathbb{R}^{N}$, which is defined by the equation

$$
\mathbf{D}_{x}^{\alpha} f(x)=\frac{1}{d_{N}(n, \alpha)} \int_{\mathbb{R}^{N}} \frac{\Delta_{y}^{n} f(x)}{|y|^{\alpha+N}} d^{N} y, \quad(n-1<\alpha<n) .
$$

To denote the Riesz fractional derivative (55), the notation $(-\Delta)^{\alpha / 2}$ is also used. Note that the Fourier transform $\mathcal{F}$ of this derivative (see Property 2.34 of Ref. [4]) is defined by

$$
\left(\mathcal{F}\left\{\mathbf{D}_{x}^{\alpha} f(x)\right\}\right)(\mathbf{k})=|\mathbf{k}|^{\alpha}(\{\mathcal{F} f(x)\})(\mathbf{k}) .
$$

The representation of the assumption in the form (52) and (53) allows us to obtain a fractional kinetic equation for arbitrary $\mathrm{N}$-dimensional space (for example, in the 3-dimensional Euclidean space).

The partial time differentiation of Eq. (49) gives

$$
\frac{\partial}{\partial t} \rho(t, x)=\varepsilon a(t) \mathbf{D}_{x}^{\alpha_{1}} \rho_{0}(x)+\varepsilon^{2} b(t, x) \mathbf{D}_{x}^{\alpha_{2}} \rho_{0}(x)+O\left(\varepsilon^{3}\right) .
$$

Substitution of Eq. (49) in the form

$$
\rho_{0}(x)=\rho(t, x)-\varepsilon \int_{0}^{t} \mathrm{~d} t_{1} a\left(t_{1}\right) \mathbf{D}_{x}^{\alpha_{1}} \rho_{0}(x)+O\left(\varepsilon^{2}\right)
$$

into Eq. (56) gives

$$
\frac{\partial}{\partial t} \rho(t, x)=\varepsilon a(t) \mathbf{D}_{x}^{\alpha_{1}} \rho(t, x)+\varepsilon^{2}\left(b(t, x) \mathbf{D}_{x}^{\alpha_{2}}-c(t) \mathbf{D}_{x}^{2 \alpha_{1}}\right) \rho(t, x)+O\left(\varepsilon^{3}\right),
$$

where

$$
c(t)=a(t) \int_{0}^{t} \mathrm{~d} t_{1} a\left(t_{1}\right)
$$

Eq. (58) up to $O\left(\varepsilon^{3}\right)$ has the form

$$
\frac{\partial}{\partial t} \rho(t, x)=\varepsilon a(t) \mathbf{D}_{x}^{\alpha_{1}} \rho(t, x)+\varepsilon^{2}\left(b(t, x) \mathbf{D}_{x}^{\alpha_{2}}-c(t) \mathbf{D}_{x}^{2 \alpha_{1}}\right) \rho(t, x)
$$

This is the fractional kinetic equation with non-integer derivatives of the order $1<\alpha_{2}<2$ and $0<2 \alpha_{1}<2$, with respect to coordinate $x$.

If $a(t)=0$, i.e.. $A\left(t, x \mid x_{1}\right)=0$, then we have the fractional equation of order $1<\alpha_{2}<2$ with respect to $x$, such that

$$
\frac{\partial}{\partial t} \rho(t, x)=\varepsilon^{2} b(t, x) \mathbf{D}_{x}^{\alpha_{2}} \rho(t, x) .
$$

This is the fractional Fokker-Planck equation that is suggested in Ref. [13] to describe fractional kinetics.

\section{Conclusion}

In this paper, we consider Fokker-Planck equations with coordinate derivatives of non-integer order $1<\alpha<2$. These derivatives are defined in the form of the fractional Marchaud and Riesz differentiations. The starting point of our consideration is the well-known Kolmogorov-Feller equation, and some generalization of the equation. The fractional kinetic equations are derived by using the method of successive approximations, and averaging with respect to a fast variable. In the paper, we assume that the correlation function of probability density for the generalized Kolmogorov-Feller equation has power-law form. Note that some properties the Fokker-Planck equation with coordinate derivatives of noninteger order $1<\alpha<2 \mathrm{~d}$ are considered in Refs. [2,8,14-18]. 


\section{References}

[1] E.W. Montroll, M.F. Shlesinger, On the wonderful world of random walks, in: J. Lebowitz, E.W. Montroll (Eds.), Studies in Statistical Mechanics, vol. 11, North-Holland, Amsterdam, 1984, p. 1.

[2] A.I. Saichev, G.M. Zaslavsky, Fractional kinetic equations: Solutions and applications, Chaos 7 (4) (1997) 753-764.

[3] S.G. Samko, A.A. Kilbas, O.I. Marichev, Fractional Integrals and Derivatives Theory and Applications, Gordon and Breach, New York, 1993.

[4] A.A. Kilbas, H.M. Srivastava, J.J. Trujillo, Theory and Application of Fractional Differential Equations, Elsevier, Amsterdam, 2006.

[5] I. Podlubny, Fractional Differential Equations, Academic Press, San Diego, 1999.

[6] A. Carpinteri, F. Mainardi, Fractals and Fractional Calculus in Continuum Mechanics, Springer, New York, 1997.

[7] Applications of Fractional Calculus in Physics, World Scientific, Singapore, 2000.

[8] G.M. Zaslavsky, Chaos, fractional kinetics, and anomalous transport, Phys. Rep. 371 (2002) 461-580.

[9] G.M. Zaslavsky, Hamiltonian Chaos and Fractional Dynamics, Oxford University Press, Oxford, 2005.

[10] J. Sabatier, O.P. Agrawal, J.A. Tenreiro Machado (Eds.), Advances in Fractional Calculus, in: Theoretical Developments and Applications in Physics and Engineering, Springer, Dordrecht, 2007.

[11] O.P. Agrawal, Fractional variational calculus in terms of Riesz fractional derivatives, J. Phys. A 40 (2007) 6287-6303; O.P. Agrawal, A general finite element formulation for fractional variational problems, J. Math. Anal. Appl. 337 (2008) 1-12.

[12] D. Baleanu, New applications of fractional variational principles, Rep. Math. Phys. 61 (2008) 199-206; D. Baleanu, J.J. Trujillo, On exact solutions of a class of fractional Euler-Lagrange equations, Nonlinear Dynamics 52 (2008) 331-335.

[13] G.M. Zaslavsky, Fractional kinetic equation for Hamiltonian chaos, Physica D 76 (1994) 110-122.

[14] G.M. Zaslavsky, Renormalization group theory of anomalous transport in systems with Hamiltonian chaos, Chaos 4 (1994) 25-33.

[15] A.V. Milovanov, Stochastic dynamics from the fractional Fokker-Planck-Kolmogorov equation: Large-scale behavior of the turbulent transport coefficient, Phys. Rev. E 63 (2001) 047301.

[16] V.V. Yanovsky, A.V. Chechkin, D. Schertzer, A.V. Tur, Levy anomalous diffusion and fractional Fokker-Planck equation, Physica A 282 (2000) 13-34.

[17] V.E. Tarasov, Fractional Fokker-Planck equation for fractal media, Chaos 15 (2005) 023102

[18] V.N. Kolokoltsov, Generalized continuous-time random walks (CTRW), subordination by hitting times and fractional dynamics, E-print: arXiv:0706. 1928.

[19] B.V. Gnedenko, The Theory of Probability, Chelsea, New York, 1962.

[20] G.M. Zaslavsky, Chaos in Dynamic Systems, Harwood Academic Publishers, New York, 1985. 